\title{
Efficacy and Feasibility of Home-Based Training for Individuals With Homonymous Visual Field Defects
}

\author{
Lina Aimola, PhD ${ }^{1,2}$, Alison R. Lane, PhD', Daniel T. Smith, PhD', \\ Georg Kerkhoff, PhD ${ }^{3}$, Gary A. Ford, FRCP ${ }^{4}$, and Thomas Schenk, PhD ${ }^{5}$
}

\begin{abstract}
Background. Homonymous visual field defects (HVFDs) are one of the most common consequences of stroke. Compensatory training encourages affected individuals to develop more efficient eye movements to improve function. However, training is typically supervised, which can be time consuming and costly. Objective. To develop and evaluate the efficacy and feasibility of an unsupervised reading and exploration computer training for individuals with HVFDs. Methods. Seventy individuals with chronic HVFDs were randomly assigned to I of 2 groups: intervention or control. The former received 35 hours of reading and exploration training, and the latter received 35 hours of control training. Visual and attentional abilities were assessed before and after training using perimetry, visual search, reading, activities of daily living, the Test of Everyday Attention, and a Sustained Attention to Response task. Results. Eighteen individuals failed to complete the training; analyses were conducted on the remaining 28 intervention and 24 control group participants. Individuals in the intervention group demonstrated significant improvements in the primary outcomes of exploration $(12.87 \%$, $95 \%$ confidence interval $[\mathrm{Cl}]=$ $8.44 \%$ to $17.30 \%)$ and reading $(18.45 \%, 95 \% \mathrm{Cl}=9.93 \%$ to $26.97 \%)$, which were significantly greater than those observed following the control intervention (exploration $=4.80 \%, 95 \% \mathrm{Cl}=0.09 \%$ to $9.51 \%$; reading $=1.95 \%, 95 \% \mathrm{Cl}=-4.78 \%$ to 8.68\%). Participants in the intervention group also reported secondary subjective improvements, although these were not matched by objective gains in tasks simulating activities of daily living. Conclusions. Home-based compensatory training is an inexpensive accessible rehabilitation option for individuals with HVFDs, which can result in objective benefits in searching and reading, as well as improving quality of life.
\end{abstract}

\section{Keywords}

hemianopia, quadrantanopia, reading, rehabilitation, transfer of training

\section{Introduction}

Homonymous visual field defects (HVFDs) are one of the most frequent consequences of brain injury.$^{1-3}$ Hemianopia (blindness in half the visual field) is the most common, followed by quadrantanopia (blindness in one quarter of the visual field). ${ }^{4}$ Individuals with HVFDs exhibit slow and inefficient exploration, ${ }^{5,6}$ are visually disoriented, and experience problems such as finding objects, avoiding obstacles, and reading. Their impairment has a significant negative impact on quality of life, ${ }^{7-9}$ and spontaneous recovery is limited. ${ }^{10,11}$

Although a variety of experimental rehabilitation techniques have been developed (including restorative, compensatory, and substitution methods; see Lane et $\mathrm{al}^{12}$ for a review), these are rarely used in standard practice, possibly because evidence for the efficacy of any technique is inconclusive. ${ }^{13}$ Compensatory training, which encourages individuals to develop more effective eye movements to cope with their visual loss, is consistently identified as the most promising. ${ }^{12-15}$ Although few studies have evaluated such therapy relative to a control intervention, several studies have reported significant benefits following compensatory training such as improved exploration, more efficient saccadic behavior, and subjective improvements in activities of daily living (ADL). ${ }^{16-23}$ Specific compensatory reading

\footnotetext{
'Durham University Queen's Campus, Stockton-on-Tees, UK

${ }^{2}$ Centre for Quality Improvement, Royal College of Psychiatrists, London, UK

${ }^{3}$ Saarland University, Saarbrücken, Germany

${ }^{4}$ Newcastle University, Newcastle, UK

${ }^{5}$ University of Erlangen-Nuremberg, Erlangen, Germany

Corresponding Author:

Alison Lane, CNRU, Wolfson Research Institute, Durham University Queen's Campus, University Boulevard, Stockton-on-Tees, TSI7 6BH, UK.

Email: a.r.lane@durham.ac.uk
} 
training has also been developed and positive effects reported. ${ }^{24-26}$

Compensatory training can be time consuming, labor intensive, and often requires specialist facilities such as large-scale training boards ${ }^{19}$ or perimeters, ${ }^{18}$ thereby limiting its availability. Even the majority of computer-based training is either completed in clinic ${ }^{16,17}$ or at home with therapist supervision, ${ }^{20,22,23}$ although one recent study has demonstrated that a Web-based reading training can increase reading speed in patients with right-sided HVFDs. ${ }^{27}$ Whether it is the patient travelling to access treatment, or the therapist travelling to supervise training, this increases the cost and time associated with rehabilitation, as well as potentially limiting access to those patients within commutable distance.

The main aim of the present controlled study was to investigate the efficacy of a new unsupervised compensatory training for individuals with HVFDs. Patients trained independently at home, limiting their exposure to the researcher, thereby controlling the influence that the therapist-patient relationship may have on rehabilitation success. Previous studies have demonstrated training specificity with regard to exploration and reading. ${ }^{22,28}$ Since these are 2 of the most frequently observed impairments in patients with HVFDs, ${ }^{4}$ it was decided that the training should incorporate elements tailored toward each skill. To our knowledge this is the first program combining both components within one rehabilitation package, meaning that individuals would only have to access and learn to use 1 tool. The primary outcomes of the study were therefore the effect of the interventions on visual exploration and reading.

Some additional secondary outcome measures were included. Since rehabilitation is aimed at improving patient activity and participation, ${ }^{29}$ transferability of the benefits to ADL was also investigated. Measures of attention were included as it is has been proposed that attention plays a role in HVFD rehabilitation. ${ }^{4,22}$ Finally, the feasibility of training delivery was estimated based on the level of acceptance, dropout rate, and demand for technical assistance.

\section{Methods}

\section{Participants}

Study approval was granted by Durham University and Northern and Yorkshire Multi-Centre Research Ethics Committees. Participants provided written informed consent in accordance with the Declaration of Helsinki. ${ }^{30}$ Seventy patients with chronic HVFDs resulting from any postchiasmatic lesion were recruited from local hospitals or as self-referrals, with visual loss confirmed using monocular automatic perimetry (Twinfield 2, Oculus Optikgerate $\mathrm{GmbH}$, Wetzlar, Germany). None had previous access to any formal HVFD rehabilitation (restoration, substitution, or compensation). Participants had to be at least 18 years old. Additional exclusion criteria included medical instability, inability to provide informed consent, visual loss as a consequence of prechiasmatic damage or a progressive condition, photosensitive epilepsy, oculomotor disorders, and severe cognitive impairment. Participants were not enrolled until at least 3 months after onset to minimize confounding by spontaneous recovery ${ }^{31}$ (range $=3-276$ months).

Participants were randomly assigned to 2 groups: intervention and control (see Table 1 for details). Some patients had additional impairments including hemiparesis $(\mathrm{n}=6)$, mild memory and cognitive impairments $(\mathrm{n}=8)$, and aphasia $(n=2)$. Where necessary visual acuity was corrected as per any existing prescription, and was not retested. Three patients ( 2 in the intervention group, 1 in the control group) had comorbid neglect as confirmed with the bells test. ${ }^{32}$ Neglect is defined as a failure to respond or orient to stimuli in contralesional space ${ }^{33}$ and can result in similar problems as HVFDs, including impaired exploration and reading. ${ }^{34}$

\section{Design}

The trial was registered on the UK Clinical Research Network Portfolio (UKCRN, ID 7144). A randomized, controlled, parallel-group design was used, comparing the effects of reading and exploration (R-E) training with a control attention-based training. Participants were randomized equally to 2 groups (R-E or control) using parallel trial allocation software ${ }^{35}$ and were advised to complete the allocated training (details below) for 1 hour per day during a time window of approximately 5 weeks. Participants were informed about the training types but did not know to which group they were assigned. Individuals in the control condition were offered the R-E training on completion. Participants completed the same assessment tasks before and after training.

\section{Materials and Procedures: Assessment}

The assessment tasks were completed in a pseudo-random order and counterbalanced across the sample. Participants completed tasks using their dominant hand, except those with hemiparesis who used their ipsilesional hand.

Perimetry. Monocular visual fields were assessed using an Oculus Twinfield 2 perimeter and the Esterman preset program, repeated for each eye. Fixation was monitored using a video-camera and central probe stimuli. One hundred target positions were assessed for each eye, with fixation accuracy (\%) and number of undetected stimuli ("misses") recorded. Forty patients also completed suprathreshold binocular kinetic perimetry, in which a white target $\left(0.25^{\circ}\right.$ in diameter $)$ moved inwards until detected at a speed of $2^{\circ}$ per second along 24 trajectories in a random order. Kinetic perimetry 


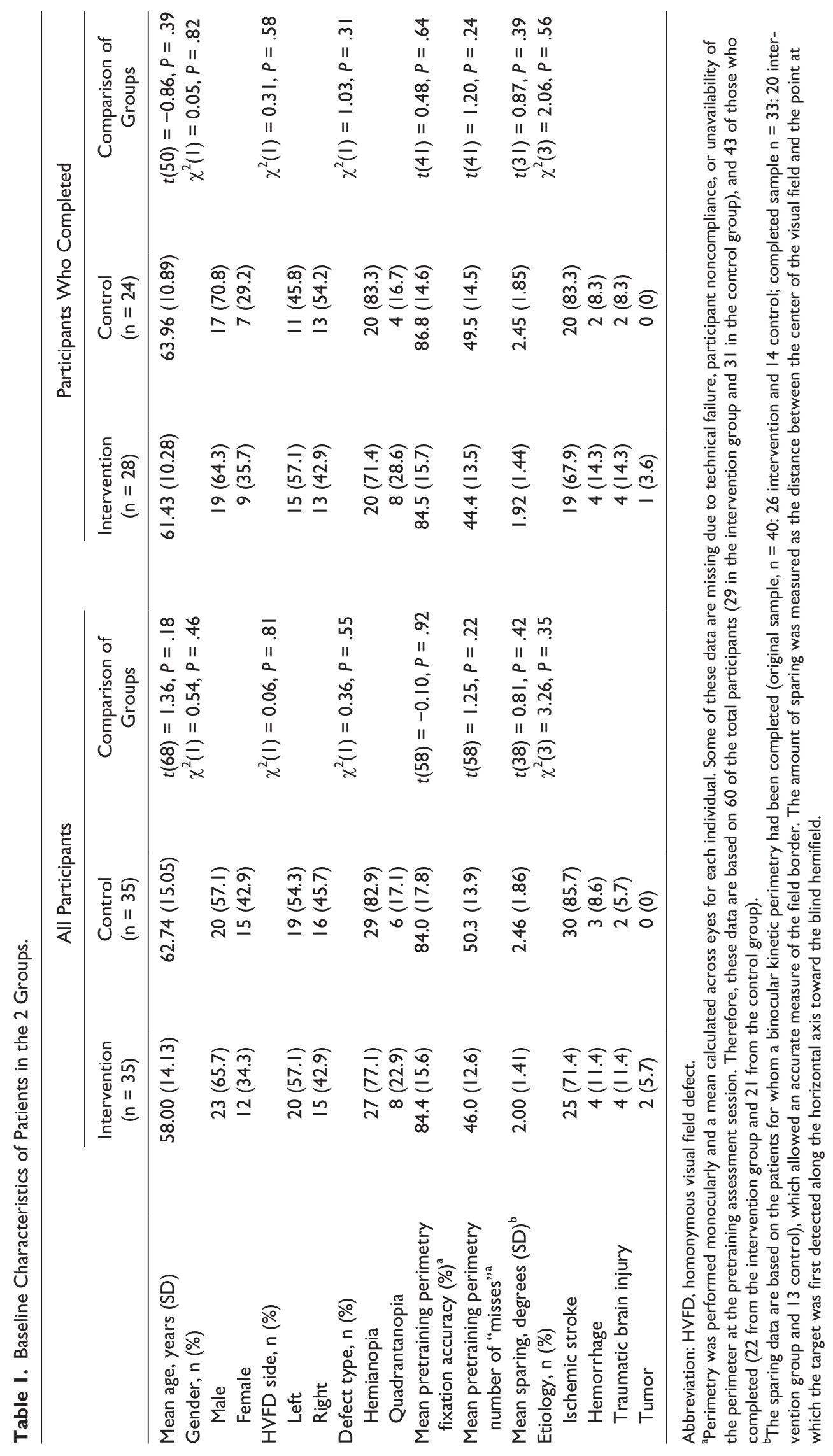


was introduced when it became clear that the spatial resolution of the Esterman finding was insufficient to provide precise characterization of the visual field border.

Visual search: Find the number. This primary outcome task (not part of training) was programmed using E-Prime 1.1 (Psychology Software Tools, Inc, Pittsburgh, PA). Participants had to scan an array (subtending approximately $47^{\circ}$ by $29^{\circ}$ of visual angle) of yellow items displayed on a black background for a specific target (numbers 1-4) among distractors (ie, $£, \#$, ?), indicating their response as quickly as possible by pressing the corresponding keyboard number. Items subtended $1^{\circ}$, with position randomized. The target appeared equally frequently in each screen quadrant. Trials began with a central white fixation cross $\left(1^{\circ}\right)$ for $1000 \mathrm{~ms}$, followed by the search array until response. There was a 1500 -ms intertrial interval; a blank black screen. Participants completed 8 practice trials and 40 test trials per session. Mean response time (RT) was calculated using correct response trials.

Reading. Reading aloud ability was assessed as another primary outcome using the same 200 word passages from a previous study, ${ }^{22}$ with reading time (seconds) and number of errors recorded. The corrected reading speed in words per minute (wpm) was calculated using the following formula: (words read - number of errors)/time $\times 60$.

\section{Tasks simulating ADL}

1. Driving hazard perception. A hazard perception task (The Studios, Rugley, 2009; www.focusmm.co.uk) was presented on a $47 \mathrm{~cm} \times 29 \mathrm{~cm}$ screen, with participants seated at a distance of $57 \mathrm{~cm}$. Participants were instructed to scan the clips and press a button on noticing potential hazards. The computer program scored responses; the earlier the hazard was noticed the more points awarded (0-5). Three practice clips were completed. The test contained 14 one-minute clips, with 10 second breaks between clips. Across 14 clips there were 16 hazards. Participants received a total score out of 80 , and a mean score per hazard was then calculated.

2. Obstacle avoidance. Participants had to walk through a corridor (15.4 m long) containing 6 obstacles. Eight paper targets (used to guide participants) were placed on the walls at a height of $165 \mathrm{~cm}$. Participants had to complete the track avoiding obstacles and removing targets. Completion time (CT) was recorded.

3. Visuomotor search. Participants stood arm's length away from a $100 \mathrm{~cm} \times 100 \mathrm{~cm}$ shelving unit, containing 5 rows $(20 \mathrm{~cm}$ in height $)$ each with $5 \mathrm{com}$ partments (width range $=10-30 \mathrm{~cm}$ ). The central compartment contained a computer mouse. Each remaining compartment contained different household items (ie, sunglasses, coffee jar) labeled with a number ( $1 \mathrm{~cm}$ tall) from 1 to 24 . Items were arranged in 1 of 3 arrays (containing nonconsecutive numbers) for each of the 5 trials. Participants had to point, in order, to 10 objects $(1-10,11-20,8-17$, $5-14,15-24)$. Each sequence was used once and the order randomized. Participants wore liquid-crystal shutter glasses. ${ }^{36}$ The experimenter used the computer mouse to clear the glasses and start the trial. When the participant pointed to the last object a second mouse-click indicated the end of the trial, turning the glasses opaque. This procedure restricted the viewing time, preventing participants memorizing the array. The time between first and second mouseclicks was the CT.

\section{Attention tasks}

1. Sustained Attention to Response (SART). ${ }^{37-39}$ Numbers 1 to 9 were sequentially presented centrally on a computer screen. Each appeared for $250 \mathrm{~ms}$ with a central fixation cross ( $900 \mathrm{~ms}$ ) between items. Numbers were white on a black background; size varied randomly between approximately $2^{\circ}$ and $5^{\circ}$ of visual angle. Participants pressed the space-bar as soon as each number appeared except number 3 (no-go trials). A total of 243 trials were performed, including 18 practice trials. The mean percentage error score on no-go trials was calculated.

2. Test of Everyday Attention (TEA)..$^{40,41}$ Three subtests were used: the visual elevator task, and the auditory elevator task both with and without distraction. Full details of these tasks can be found in Robertson et al. ${ }^{40}$

Subjective Questionnaires. The Visual Functioning Questionnaire (VFQ-25) ${ }^{42}$ and Visual Impairments Questionnaire (VIQ) ${ }^{17,22}$ both involved participants rating their difficulty in carrying out specific activities (ie, crossing the street, reading). The Subjective Reasons Questionnaire (SRQ; Supplementary Material A; available online at http://NNR. sagepub.com/supplemental) was used to evaluate whether patients thought the training helped them, and if so, which aspect they found most beneficial. The extent to which they agreed with statements relating to why they think their condition improved, what aspect of training was most helpful, and which part of the assessment was most helpful was rated out of 5 (with higher scores indicating more benefit). The SRQ was administrated at the end of the study to all patients who completed the R-E training, including those who completed it after the control intervention $(\mathrm{N}=46)$.

\section{Materials and Procedures: Training}

At the start of the intervention period the experimenter demonstrated the training, during which the computer was 
set up centrally at a distance of approximately $57 \mathrm{~cm}$. Participants were encouraged to train in a similar manner, although adherence cannot be guaranteed. For full details of the training, see Supplementary Material B (available online at http://NNR.sagepub.com/supplemental).

Experimental Intervention. The experimental training consisted of reading and exploration components; patients completed components sequentially with order randomized. In the visual exploration tasks patients had to find a target defined by a specific feature (color, shape, size) among an array containing distractors (ie, a red letter among blue ones). In the reading task, patients had to detect a nonword target (ie, sowels) among a varying number of words (ie, accent), presented in a single central horizontal line. In both task types participants responded to target presence using an appropriate computer-mouse press. Computer feedback on speed and accuracy of responses, and overall progress to date (ie, difficulty level achieved and number of training sessions completed), was provided at the end of each block of trials.

For both training components difficulty level was dynamically adjusted based on both accuracy and speed of previous performance. If patients were $\geq 90 \%$ accurate then difficulty would increase to the next level, whereas with accuracy $<75 \%$ difficulty would drop to an easier level. In exploration tasks difficulty was increased by enlarging the spatial zone within which a target could appear and by making targets and distractors more similar. For the reading task the word length and number of distractor words increased (up to a maximum of 7). For both tasks, presentation time was directly related to previous response times, that is, the faster the participant, the shorter the successive presentation time.

Patients could perform a maximum of 14 blocks per day. Each block contained 120 trials. They completed 294 exploration and 196 reading blocks.

Control. This training consisted of a number of tasks requiring visual attention but no systematic exploration or large horizontal eye movements. The randomly presented tasks included a Go/No-Go task, centrally presented sequential search, Sternberg task, and a "rabbit hunting" task. Difficulty was adjusted dynamically depending on performance by reducing presentation time or increasing attentional load. Patients were instructed to complete 10 blocks per day in approximately 1 hour, with a total of 350 blocks.

\section{Statistical Analyses}

Mixed-model ANOVAs were used, with the within-subject variable Session (pre- vs posttraining) and betweensubject variable Group (intervention vs control). Where relevant, paired-samples $t$ tests were used to further investigate relationships. Questionnaire data were analyzed nonparametrically (Mann-Whitney $U$ for betweensubjects and Wilcoxon signed-ranks for within-subjects comparisons). Bonferroni corrections were applied throughout as appropriate.

\section{Results}

Eighteen participants dropped out during the intervention period because of health problems (7), death (2), or low motivation (9). The final sample included in analyses consisted of 52 participants; 28 intervention and 24 control (see Figure 1).

\section{Visual Search: Find the Number}

Mean accuracy for both groups was above $96 \%$ in all conditions. ANOVA performed on RT revealed a main effect of Session, $F(1,50)=25.56, P<.01$, but not Group, $F(1,50)$ $=1.95, P=.17$. There was a significant Group by Session interaction, $F(1,50)=4.71, P<.05$; the intervention group improved significantly more than the controls (Figure 2A). The mean change in RT for the intervention group was $12.87 \%(95 \%$ confidence interval $[\mathrm{CI}]=8.44 \%$ to $17.30 \%)$, and only $4.80 \%$ for the control group $(95 \% \mathrm{CI}=0.09 \%$ to 9.51\%).

\section{Reading}

A significant difference was observed in the pretraining reading speed of individuals with left and right HVFDs, $t(50)=13.43, P=.001$; those with a right-sided HVFD were significantly slower. An ANOVA was performed including a factor for side of defect (Hemifield). This revealed a significant effect of Session, $F(1,50)=6.88, P=$ .01 ; participants read significantly faster after training (Figure 2B). The significant interaction between Group and Session, $F(1,50)=9.01, P<.01$, showed that improvement observed for the intervention group $(18.45 \%, 95 \% \mathrm{CI}=$ $9.93 \%$ to $26.97 \%$ ) was larger than that of the controls $(1.95 \%, 95 \% \mathrm{CI}=-4.78 \%$ to $8.68 \%)$. No significant Group effect was observed, $F(1,50)=1.01, P=.32$. Furthermore, there were no significant interactions involving the factor Hemifield $(P \geq .33)$, demonstrating that reading speed increased following experimental intervention regardless of defect side (Table 2).

\section{Tasks Simulating ADL}

Visuomotor Search. Three intervention group patients were unable to perform this task so were excluded from the analysis. ANOVA performed on CT revealed a significant effect of Session, $F(1,47)=5.19, P<.05$ (Table 3 ), with patients faster after training. There was no significant effect 


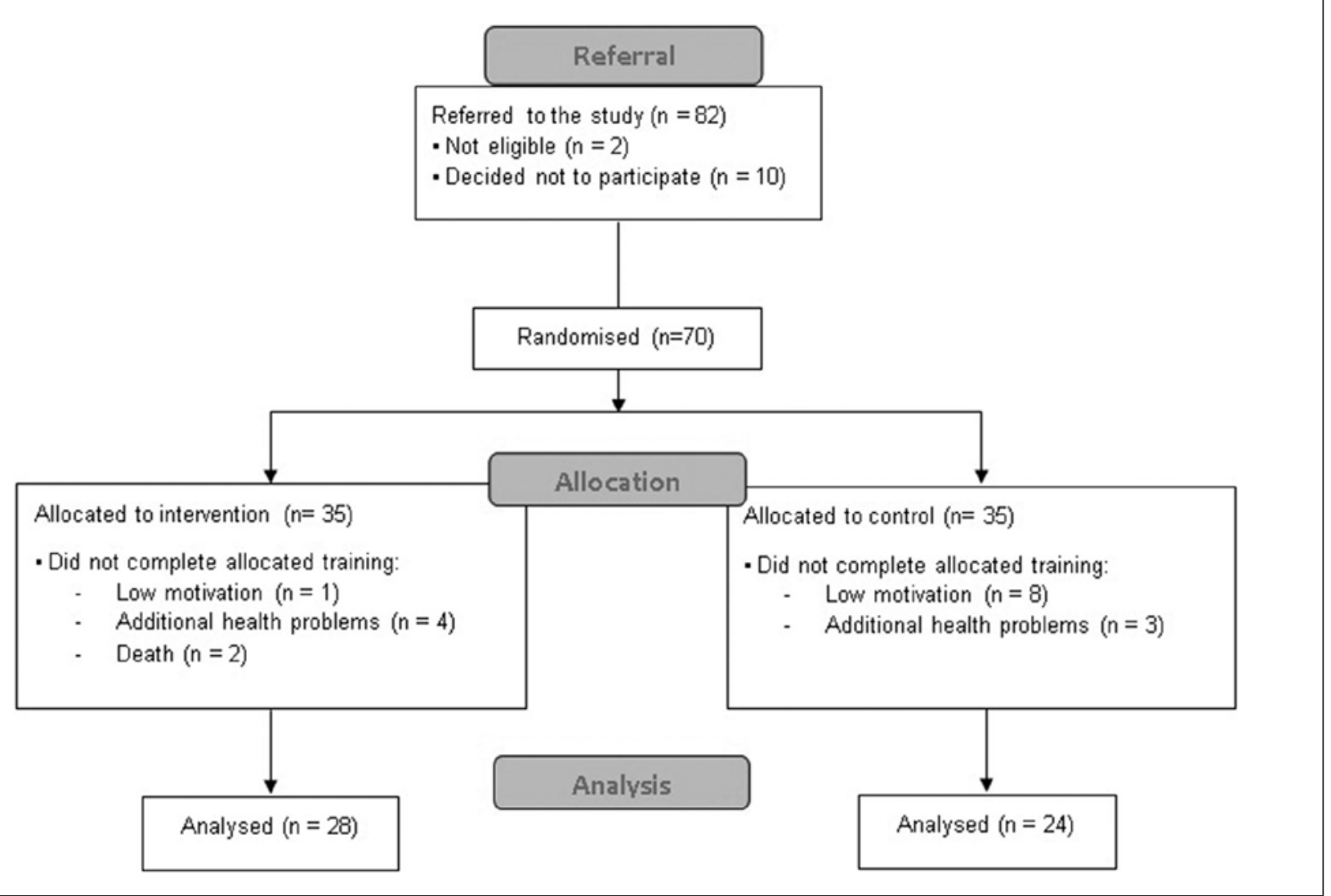

Figure I. CONSORT participant flow diagram.

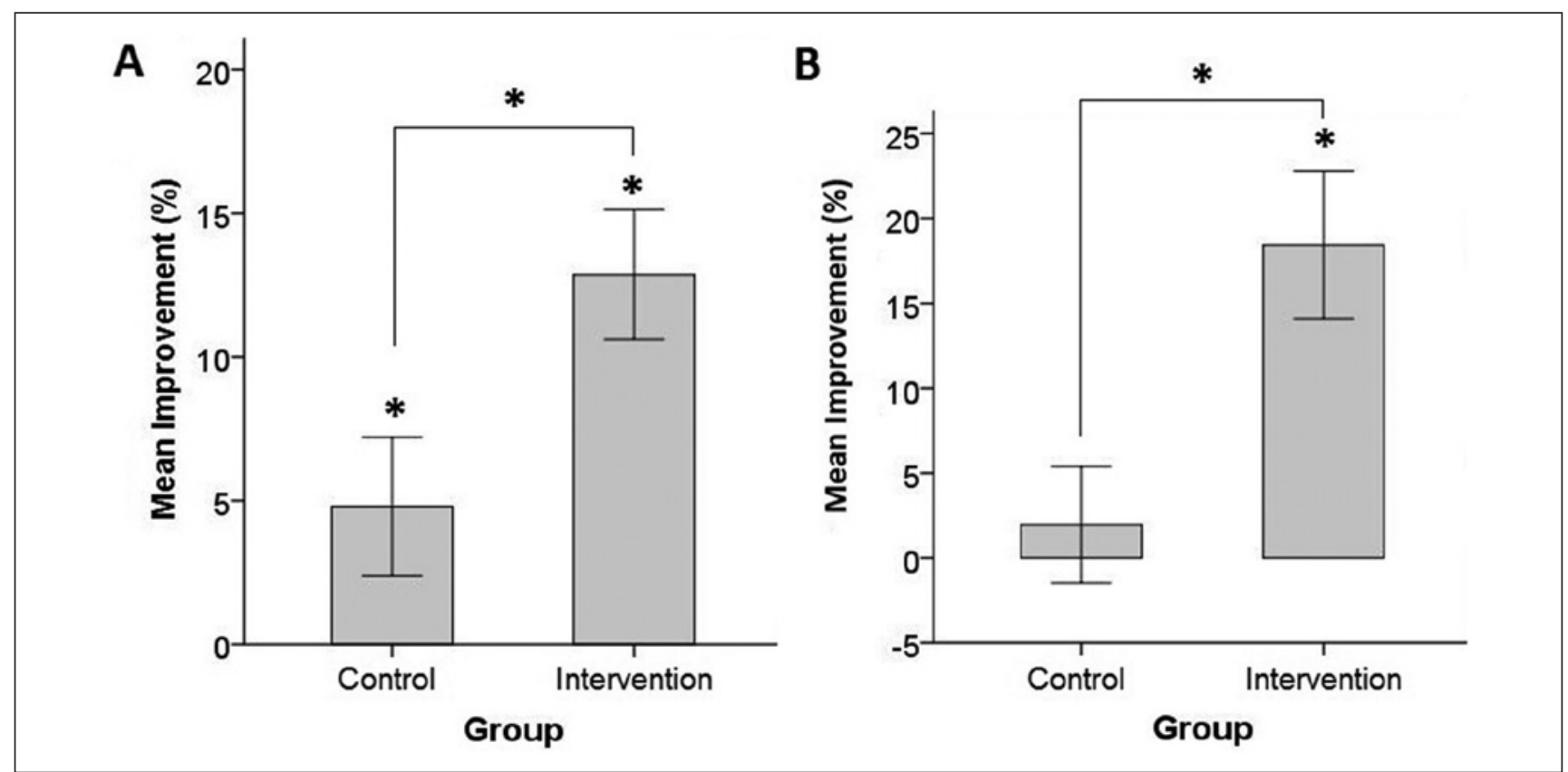

Figure 2. Bar-graphs showing the mean ( \pm I standard error) percentage improvement in performance at the posttraining session relative to the pretraining session, for the intervention and control groups, for the find the number visual search task $(A)$ and the reading task (B). Significant differences are shown $(*)$. 
of Group, $F(1,47)=0.11, P=.74$, or Group and Session interaction, $F(1,47)=0.09, P=.76$.

Obstacle Avoidance. Four patients were unable to complete this task ( 3 in the intervention group and 1 in the control group) so were excluded from the analysis. The ANOVA showed no significant main effects or interactions ( $P \geq .09$; Table 3$)$, indicating no change in CT after either training.

Table 2. Mean Corrected Reading Speed in Words per Minute (With Standard Deviation) Both Before and After Training for the 2 Groups, Separated Into Those With a Left or Right Visual Field Defect. ${ }^{2}$

\begin{tabular}{lcc}
\hline & \multicolumn{2}{c}{$\begin{array}{c}\text { Mean Corrected Reading Speed } \\
\text { (wpm) }\end{array}$} \\
\cline { 2 - 3 } & Pretraining & Posttraining \\
\hline Intervention group (n) & $114.43(42.19)$ & $132.46(50.16)^{*}$ \\
Left (I5) & $136.20(23.85)$ & $157.73(34.82)^{*}$ \\
Right (I3) & $89.31(45.44)$ & $103.31(50.28)^{*}$ \\
Control group (n) & $110.33(56.38)$ & $109.13(52.63)$ \\
Left (I I) & $132.82(48.94)$ & $128.73(41.85)$ \\
Right (I3) & $91.31(56.92)$ & $92.54(56.56)$ \\
\hline
\end{tabular}

${ }^{\text {a }}$ Statistically significant changes between pre- and posttraining assessments are indicated $(*)$.
Driving and Hazard Perception. Analysis of the mean score revealed no significant main effects or interactions $(P \geq .25$; Table 3), indicating no improvement in hazard perception.

\section{Attention Tasks}

SART. The ANOVA performed on the mean error rate revealed no significant main effects or interactions $(P \geq .09$; Table 3), indicating unchanged sustained attention.

TEA. Two patients (1 patient in each group) were unable to perform the visual elevator task, so were excluded from the analysis, while 1 intervention group patient did not complete the auditory version.

TEA: Visual elevator. The ANOVA revealed a main effect of Session on accuracy, $F(1,48)=16.69, P<.01$ (Table 3), but no effect of Group, $F(1,48)=1.61, P=.21$, and no interaction between Group and Session, $F(1,48)=2.13, P=.15$. Analysis of the time per switch component revealed a significant Session effect, $F(1,48)=7.11, P=.01$. There was no effect of Group, $F(1,48)=2.32, P=.13$, and no interaction, $F(1,48)=1.62, P=.21$

TEA: Auditory elevator without distraction. The ANOVA performed on mean accuracy scores revealed no significant effects or interactions $(P \geq .31$; Table 3$)$, indicating that performance remained unchanged.

Table 3. Mean Results (With Standard Deviation) for Each of the Tasks Simulating Activities to Daily Living and the Attention Tasks, for the Intervention and Control Groups and Each Assessment Session Separately.

\begin{tabular}{|c|c|c|c|c|c|c|}
\hline & \multicolumn{3}{|c|}{ Intervention Group } & \multicolumn{3}{|c|}{ Control Group } \\
\hline & $\mathrm{n}$ & Pretraining & Posttraining & $\mathrm{n}$ & Pretraining & Posttraining \\
\hline \multicolumn{7}{|c|}{ ADL } \\
\hline Obstacles $^{\mathrm{a}}$ & 25 & $40(25)$ & $35(19)$ & 23 & $44(14)$ & $45(19)$ \\
\hline Visuomotor search ${ }^{\mathrm{a}}$ & 25 & II4 (5I) & $101(53)$ & 24 & 117 (49) & $107(40)$ \\
\hline Driving simulator ${ }^{\mathrm{b}}$ & 28 & $2.9(0.76)$ & $2.9(0.84)$ & 24 & $2.7(0.50)$ & $2.9(0.7 \mathrm{I})$ \\
\hline \multicolumn{7}{|l|}{ Attention } \\
\hline \multicolumn{7}{|l|}{ TEA } \\
\hline Visual elevator & 27 & & & 23 & & \\
\hline Accuracy (\%) & & $7.9(2.32)$ & $8.8(1.61)$ & & $6.8(2.47)$ & $8.7(2.11)$ \\
\hline Time per switch ${ }^{c}$ & & $5.5(2.07)$ & $4.5(1.28)$ & & $5.9(2.53)$ & $5.6(2.94)$ \\
\hline Auditory elevator & 27 & & & 24 & & \\
\hline No distraction ${ }^{d}$ & & $6.7(0.81)$ & $6.8(0.64)$ & & $6.6(0.83)$ & $6.5(0.78)$ \\
\hline With distraction ${ }^{d}$ & & $8.0(2.69)$ & $7.5(2.83)$ & & $7.7(2.82)$ & $7.9(3.35)$ \\
\hline SART ${ }^{\mathrm{e}}$ & 28 & $17.4(17.80)$ & $9.9(14.62)$ & 24 & $17.8(15.65)$ & $19.2(20.43)$ \\
\hline
\end{tabular}

Abbreviations: ADL, activities of daily living; TEA, Test of Everyday Attention; SART, Sustained Attention to Response.

${ }^{a}$ Seconds (SD).

bMean accuracy score (SD).

'Calculation described in the Methods section (SD).

'Mean accuracy score (SD).

${ }^{\mathrm{e}}$ Percentage error (SD). 
Table 4. Mean Rating (SD) for Each Item of the Visual Impairments Questionnaire (VIQ) and the Visual Functioning Questionnaire (VFQ-25), for Each Assessment Session for the 2 Groups. $^{a}$

\begin{tabular}{|c|c|c|c|c|}
\hline & \multicolumn{2}{|c|}{ Intervention Group $(\mathrm{n}=28)$} & \multicolumn{2}{|c|}{ Control group $(n=24)$} \\
\hline & Pretraining & Posttraining & Pretraining & Posttraining \\
\hline \multicolumn{5}{|l|}{ VIQ } \\
\hline Seeing objects & $2.18(1.09)$ & $\mathrm{I} .57(0.88) *$ & $2.67(1.17)$ & $2.58(1.18)$ \\
\hline Bumping into obstacles & 1.64 (I.39) & $0.89(1.10)^{*}$ & $1.92(1.53)$ & I.7I (1.40) \\
\hline Loosing way & $\mathrm{I} .57(\mathrm{I} .50)$ & $1.18(1.39)^{*}$ & 1.30 (I.49) & $\mathrm{I} .04(1.30)$ \\
\hline Finding items (table) & $1.25(1.14)$ & $0.89(1.10)^{*}$ & I. $17(0.96)$ & $1.17(0.96)$ \\
\hline Finding items (room) & I.7I (I.05) & $1.14(1.14)^{*}$ & $\mathrm{I} .83(1.24)$ & 1.75 (1.19) \\
\hline Finding items (shop) & $2.11(1.34)$ & $1.28(1.30)^{*}$ & $2.37(1.17)$ & $2.21(1.14)$ \\
\hline Using public transport & $1.36(1.52)$ & $\mathrm{I} .2 \mathrm{I}(\mathrm{I} .55)$ & $1.66(1.58)$ & $\mathrm{I} .50(\mathrm{I} .53)$ \\
\hline Find way at home & $0.50(1.07)$ & $0.32(0.90)$ & $0.37(0.9)$ & $0.37(0.92)$ \\
\hline Crossing the street & I.7I (I.44) & $1.28(1.56)^{*}$ & I.87 (1.39) & $\mathrm{I} .83$ ( 1.52$)$ \\
\hline Reading & $1.93(1.49)$ & $\mathrm{I} .2 \mathrm{I}(\mathrm{I} .34)^{*}$ & $2.17(1.58)$ & $2.17(1.63)$ \\
\hline \multicolumn{5}{|l|}{ VFQ-25 } \\
\hline General vision & $3.03(0.79)$ & $2.6 \mathrm{I}(0.74)^{*}$ & $3.08(0.65)$ & $3.12(0.80)$ \\
\hline Worry & $3.03(1.14)$ & $2.46(1.14)^{*}$ & $2.54(1.28)$ & $2.37(1.34)$ \\
\hline Reading & $2.46(1.32)$ & $1.96(1.23)^{*}$ & $2.58(I .4 I)$ & $2.50(I .4 I)$ \\
\hline Do small jobs & $3.00(1.25)$ & $2.64(1.28)^{*}$ & $2.87(1.45)$ & $2.46(1.35)^{*}$ \\
\hline Street signs & $1.68(0.94)$ & $\mathrm{I} .46(0.84)$ & $1.79(1.18)$ & $\mathrm{I} .83$ (1.27) \\
\hline Searching & $2.68(0.77)$ & $2.00(0.72)^{*}$ & $2.58(0.97)$ & $2.50(1.02)$ \\
\hline Miss objects & $2.86(0.80)$ & $2.32(0.94)^{*}$ & $2.92(1.10)$ & $2.92(1.14)$ \\
\hline Visiting friends & $2.03(1.26)$ & I.7I (1.0I) & I.67 (I.05) & $\mathrm{I} .67(0.82)$ \\
\hline Self-care & $1.68(1.02)$ & $1.61(0.92)$ & $\mathrm{I} .46(0.78)$ & $\mathrm{I} .37$ (0.77) \\
\hline Recognizing people & $1.64(1.03)$ & $1.50(1.00)$ & $1.58(0.83)$ & I.54 (0.72) \\
\hline Watching TV & I.7I (0.97) & $1.57(0.96)$ & $1.50(0.93)$ & I.54 (0.93) \\
\hline Mood change & $2.50(1.52)$ & $2.93(1.54)^{*}$ & $2.75(1.70)$ & $3.17(1.58)$ \\
\hline Going out alone & $3.18(1.59)$ & $3.57(1.62)^{*}$ & $3.00(1.74)$ & $3.29(1.57)$ \\
\hline Accomplishment & $2.86(1.01)$ & $3.14(0.89)$ & 2.71 (1.27) & $2.87(1.33)$ \\
\hline Time length working & $2.93(1.24)$ & $3.32(I .3 \mathrm{I})^{*}$ & $3.00(1.64)$ & $3.12(1.54)$ \\
\hline Staying at home & $3.68(1.61)$ & $4.00(I .4 I)$ & $4.29(1.16)$ & $4.33(1.05)$ \\
\hline Control & $2.40(1.26)$ & $2.89(1.34)^{*}$ & 2.71 (1.49) & $2.79(1.44)$ \\
\hline Help from others & $3.14(1.43)$ & $3.35(1.43)$ & $3.29(1.46)$ & 3.37 (1.47) \\
\hline
\end{tabular}

a Lower scores are associated with less impairment, except the last 5 items of the VFQ-25 where a higher score indicates lower impairment. Significant differences are shown $(*)$.

TEA: Auditory elevator with distraction. There were no significant main effects or interactions revealed by the ANOVA $(P \geq .27$; Table 3).

\section{Questionnaires}

For both the VIQ and the VFQ-25, the majority of items showed a significant improvement (lower mean rating) after the R-E intervention $(U \geq 149.5 ; P<.05$; Table 4$)$. As for the control training, only 1 item on the VFQ-25 ("doing small jobs") showed a posttraining improvement.

\section{Training Results}

Training data were examined to determine average performance. Data were unavailable from 1 control group participant (technical failure). For the control training, the maximum training level of 12 was obtained by $83 \%(n=19)$ of patients (mean $=11.5, \mathrm{SD}=1.5)$. Mean accuracy at training completion was $90.5 \%(\mathrm{SD}=9.0)$. For the experimental training, the maximum level for the exploration component was 8 , achieved by $50 \%(n=14)$ of patients. The mean level obtained was $6.0(\mathrm{SD}=2.4)$ and mean end accuracy was $87.2 \%$ ( $\mathrm{SD}=7.9 \%)$. For the reading component the maximum level was 26 , obtained by $18 \%$ of patients $(n=5)$. The mean level achieved was $16.4(\mathrm{SD}=8.0)$, and accuracy was $85.0 \%(\mathrm{SD}=6.0)$.

Training Acceptance and Feasibility. Figure 3 illustrates the mean SRQ scores for the different questions. In particular, patients agreed that their improvements related to concentration, awareness of their condition, and 


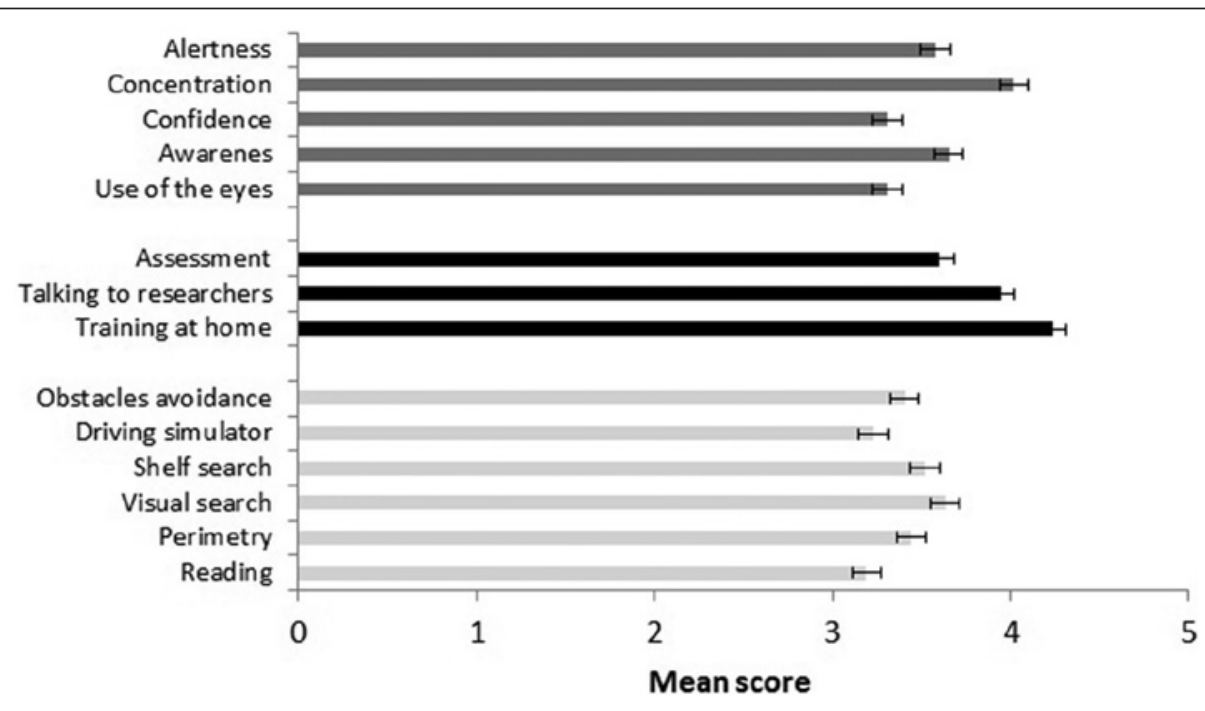

Figure 3. Bar-graph showing the mean satisfaction scores (with standard error bars) for the different questions of the SRQ subdivided into 3 categories: "why patients thought their condition improved" (dark grey bars), "aspect of the training that was most helpful to them" (black bars), and "part of the assessment they thought was most useful" (light gray bars). Higher scores indicate greater agreement.

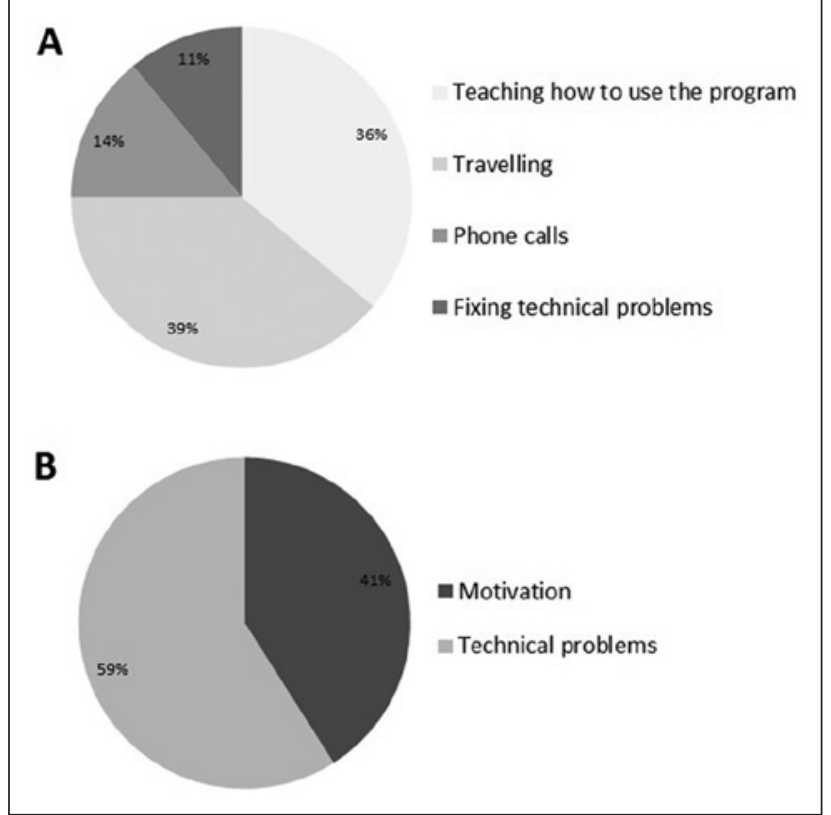

Figure 4. Pie-charts showing the percentage of time cost allocated to teaching the patients to use the program, travelling to their homes, phone calls, and fixing technical problems (A); the percentage of phone calls made to provide technical support and to maintain motivation (B).

alertness. Participants were also particularly pleased about being able to train at home. The assessment tasks which they felt helped them the most were visual and visuomotor search.
Two factors were considered to estimate the feasibility of the R-E training: compliance and support time.

Compliance. There were 18 withdrawals, 9 due to low motivation. A total of $89 \%$ of the low-motivation withdrawals were in the control group. Recommended training duration was 5 weeks. However, only 3 patients met this requirement; the mean completion time for the experimental training was 9.3 weeks $(\mathrm{SD}=6.0)$.

Support time. The time spent supporting each participant during training was recorded. The following aspects were considered: teaching patients how to use the program, travelling to their home (first visit and additional visits when needed), solving technical problems, and phone calls (differentiated between providing technical assistance or maintaining motivation; Figure 4). The mean overall support time per completed training was 137 minutes $(\mathrm{SD}=91)$.

\section{Discussion}

\section{Overview of Findings}

R-E training significantly improved exploration and reading performance relative to the control training. Patients also reported subjective improvements in several ADL domains that were specific to the R-E intervention. However, these subjective improvements were not accompanied by improvements in objective ADL tasks (obstacle avoidance, visuomotor search, and hazard perception). We 
speculate that this null result occurred because performance was measured on a small number of trials due to task length. It is therefore possible that analyses lacked the statistical power to detect small improvements. Moreover, the tasks were complex, requiring skills beyond the eye-movement strategies specifically trained.

Both R-E and control training led to significant improvements in the Visual Elevator task, but not to other measures of attention. This confirms that R-E training improves visual attention. However, the effect is no bigger than observed for other attention-training regimes and therefore cannot explain the superior effects of this training on search and reading. This implies that R-E training has a nonspecific effect (on attention) in addition to specific effects on exploration and reading. In summary, it appears that R-E training is clinically effective and specific.

To assess the feasibility of the training, we examined adherence, satisfaction, and support time. Eighteen out of $70(26 \%)$ patients failed to complete the training, half of these stopped because of health problems or death. Motivation problems accounted for only 9 dropouts, and most important, only 1 of those patients was in the experimental group. However, only 3 out of 28 patients completed the R-E training within the prescribed 5 weeks, with average completion taking 9.3 weeks (ie, almost twice as long as expected). Therefore, training can achieve satisfactory adherence but there are issues with ensuring patients keep to the treatment schedule. Satisfaction with R-E training was quite high and patients particularly liked the fact that they could train themselves at home. Regarding support time, the therapist had to spend an average of 137 minutes on each patient. This compares favorably with a typical time investment of 600 minutes ( 15 training sessions of at least 40 minutes each) for similar supervised training. ${ }^{22}$ Encouragingly, the majority of problems could be solved via phone without requiring a home visit.

\section{Comparing Our Study to Previous Findings}

Our study confirmed previous findings on the effectiveness of supervised compensatory training, ${ }^{16-26}$ and is the first to demonstrate that reading and exploration skills can be improved following unsupervised training. This finding is of obvious practical relevance, but also of theoretical interest. While previous studies ${ }^{22,28}$ demonstrated little transfer between reading and exploration (ie, exploration training does not improve reading and vice versa) it remained unclear which difference between the 2 training types was responsible for this. In our case the reading and exploration training components are very similar. Both are tasks that could be described as visual search (patients have to indicate the presence of a given target). The main difference seems to be that the reading task requires a specific search direction (horizontal, left-to-right) whereas exploration does not. It seems likely that it is the more specific oculomotor strategy which leads to the significant improvements in reading.

Second, we included a control training group. This comparison of the specific with a nonspecific (ie, attentional) training sets our study apart from most other clinical studies on the subject, with 2 exceptions. We have previously compared the efficacy of supervised exploration training with supervised attentional training, ${ }^{22}$ and Spitzyna and colleagues ${ }^{25}$ reported specific benefits of supervised optokinetic training for improving reading performance of patients with HVFDs compared to a control training. However, neither of the studies looked at the effect of combining exploration and reading training as reported here.

Interestingly, in a previous trial of exploration training, ${ }^{22}$ we found no significant improvement of reading, whereas the current study did. This shows that adding a specific reading training is sufficient and necessary to improve the reading of patients with HVFDs. However, in other respects the supervised training previously studied seemed more effective. Specifically, improvement in an untrained visual search task was substantially larger in the previous $(21.6 \%)$ than the current study (12.9\%). Numerous factors might explain this. First, the task was not entirely the same in both studies and perhaps the earlier version was more sensitive to improvements. Second, although total training time was longer in the current study since it combined 2 training components (1500 vs 600 minutes), it was also spread across a longer time interval (mean 9.3 weeks vs 4.0 weeks), which may have reduced training intensity and thus efficacy. Third, it is possible that reading and exploration components might interfere, such that the oculomotor patterns learned during the reading training disrupt the consolidation of the eye-movement skills acquired during exploration training. Finally, automated feedback provided by the unsupervised training may be less effective than human feedback. Lack of personal supervision might reduce motivation, feedback value, and thus overall efficacy.

\section{Conclusions}

The central finding is that unsupervised home-based R-E training significantly improves the primary outcomes of visual search and reading, outperforming generic attentional training. Furthermore, this training is popular with patients, achieves satisfactory adherence, and of additional importance, the time which a therapist needs to invest is 5 times less than for comparable supervised training. The lesser investment from the therapist means that implementation costs are reduced, and access can be increased because rehabilitation can be provided to more individuals simultaneously. However, this training has some drawbacks. Specifically, subjective everyday improvements were not accompanied by objective ADL-related task 
improvements. Furthermore, the unsupervised R-E training may be less effective than the supervised equivalent. Given the great potential of offering neurorehabilitative training in an unsupervised form, research on factors that might allow similar efficacy with unsupervised as with supervised training should be a high priority.

\section{Acknowledgments}

The authors would like to thank staff from local NHS trusts (including Newcastle, South Tees, City of Sunderland, and County Durham and Darlington), in addition to Action for Blind People for their assistance with participant recruitment.

\section{Authors' Note}

This article presents independent research funded by the National Institute for Health Research (NIHR) under its Research for Patient Benefit (RfPB) Programme (Grant Reference Number PB-PG-0407-12038). The views expressed are those of the author(s) and not necessarily those of the NHS, the NIHR, or the Department of Health.

\section{Declaration of Conflicting Interests}

The author(s) declared no potential conflicts of interest with respect to the research, authorship, and/or publication of this article.

\section{Funding}

The author(s) disclosed receipt of the following financial support for the research, authorship, and/or publication of this article: The project was supported by a National Institute for Health Research grant (PB-PG-0407-12038) and was adopted and supported by the North East NIHR Stroke Research Network. GAF is supported by an NIHR Senior Investigator award.

\section{References}

1. Gilhotra JS, Mitchell P, Healey PR, Cumming RG, Currie J. Homonymous visual field defects and stroke in an older population. Stroke. 2002;33:2417-2420.

2. Schlageter K, Gray B, Hall K, Shaw R, Sammet R. Incidence and treatment of visual dysfunction in traumatic brain injury. Brain Inj. 1993;7:439-448.

3. Ali M, Hazelton C, Lyden P, Pollock A, Brady M. Recovery from poststroke visual impairment: evidence from a clinical trials resource. Neurorehabil Neural Repair. 2013;27:133141.

4. Zihl J. Rehabilitation of Visual Disorders After Brain Injury. 2nd ed. Hove, UK: Psychology Press; 2011.

5. Zihl J. Visual scanning behavior in patients with homonymous hemianopia. Neuropsychologia. 1995;33:287-303.

6. Pambakian ALM, Wooding DS, Patel N, Morland AB, Kennard C, Mannan SK. Scanning the visual world: a study of patients with homonymous hemianopia. J Neurol Neurosurg Psychiatry. 2000;69:751-759.

7. Papageorgiou E, Hardiess G, Schaeffel F, et al. Assessment of vision-related quality of life in patients with homonymous visual field defects. Graefes Arch Clin Exp Ophthalmol. 2007;245:1749-1758.

8. Gall C, Lucklum J, Sabel BA, Franke GH. Vision- and health-related quality of life in patients with visual field loss after postchiasmatic lesions. Invest Ophthalmol Vis Sci. 2009;50:2765-2776.

9. Warren M. Pilot study on activities of daily living limitations in adults with hemianopsia. Am J Occup Ther. 2009;63:626-633.

10. Zihl J, Kennard C. Disorders of higher visual function. In: Brandt T, Caplan L, Dichgans JEA, eds. Neurological Disorders: Course and Treatment. San Francisco, CA: Academic Press; 1996.

11. Zhang X, Kedar S, Lynn MJ, Newman NJ, Biousse V. Natural history of homonymous hemianopia. Neurology. 2006;66:901-905.

12. Lane AR, Smith DT, Schenk T. Review of clinical treatment options for patients with homonymous visual field defects. Clin Ophthalmol. 2008;2:93-102.

13. Pollock A, Hazelton $\mathrm{C}$, Henderson $\mathrm{CA}$, et al. Interventions for visual field defects in patients with stroke. Stroke. 2012;43:e37-e38.

14. Bouwmeester L, Heutink J, Lucas C. The effect of visual training for patients with visual field defects due to brain damage: a systematic review. J Neurol Neurosurg Psychiatry. 2007;78:555-564.

15. Gold DR, Grover LL. Treatment of homonymous visual field defects. Curr Treat Options Neurol. 2012;14:73-83.

16. Kerkhoff G, Münßinger U, Haaf E, Eberle-Strauss G, Stögerer E. Rehabilitation of homonymous scotomata in patients with postgeniculate damage of the visual system: saccadic compensation training. Restor Neurol Neurosci. 1992;4:245-254.

17. Kerkhoff G, Münßinger U, Meier EK. Neurovisual rehabilitation in cerebral blindness. Arch Neurol. 1994;51:474-481.

18. Zihl J. Visual scanning behaviour in patients with homonymous hemianopia. Neuropsychologia. 1995;33:287-303.

19. Nelles G, Esser J, Eckstein A, Tiede A, Gerhard H, Diener H. Compensatory visual field training for patients with hemianopia after stroke. Neurosci Lett. 2001;306:189-192.

20. Pambakian ALM, Mannan SK, Hodgson TL, Kennard C. Saccadic visual search training: a treatment for patients with homonymous hemianopia. J Neurol Neurosurg Psychiatry. 2004;75:1443-1448.

21. Roth T, Sokolov AN, Messias A, Roth P, Weller M, Trauzettel-Klosinski S. Comparing explorative saccade and flicker training in hemianopia: a randomized controlled study. Neurology. 2009;72:324-331.

22. Lane AR, Smith DT, Ellison A, Schenk T. Visual exploration training is no better than attention training for treating hemianopia. Brain. 2010;133:1717-1728.

23. Mannan SK, Pambakian ALM, Kennard C. Compensatory strategies following visual search training in patients with homonymous hemianopia: an eye movement study. J Neurol. 2010;257:1812-1821.

24. Kerkhoff G, Münßinger U, Eberle-Strauss G, Stögerer E. Rehabilitation of hemianopic alexia in patients with postgeniculate visual field disorders. Neuropsychol Rehabil. 1992;2:21-42. 
25. Spitzyna GA, Wise RJS, McDonald SA, et al. Optokinetic therapy improves text reading in patients with hemianopic alexia: a controlled trial. Neurology. 2007;68:1922-1930.

26. Schuett S, Heywood CA, Kentridge RW, Zihl J. Rehabilitation of hemianopic dyslexia: are words necessary for re-learning oculomotor control? Brain. 2008;131:3156-3168.

27. Ong YH, Brown MM, Robinson P, Plant GT, Husain M, Leff AP. Read-Right: a "web app" that improves reading speeds in patients with hemianopia. J Neurol. 2012;259:2611-2615.

28. Schuett S, Heywood CA, Kentridge RW, Dauner R, Zihl J. Rehabilitation of reading and visual exploration in visual field disorders: transfer or specificity? Brain. 2012;135:912-921.

29. Sohlberg MM, Mateer CA. Cognitive Rehabilitation: An Integrative Neuropsychological Approach. New York, NY: Guildford Press; 2001.

30. International Committee of Medical Journal Editors. Statements from the Vancouver Group. BMJ. 1991;302:1194.

31. Pambakian ALM, Kennard C. Can visual function be restored in patients with homonymous hemianopia? Br J Ophthalmol. 1997;81:324-328.

32. Gauthier L, Dehaut F, Joanette Y. The bells test: a quantitative and qualitative test for visual neglect. Int J Clin Neuropsychol. 1989;11:49-54.

33. Heilman KM, Watson RT, Valenstein E. Neglect and related disorders. In: Heilman KM, Valenstein E, eds. Clinical Neuropsychology. 2nd ed. New York, NY: Oxford University Press; 1985.
34. Robertson IH, Marshall J. Unilateral Spatial Neglect. Hove, UK: Lawrence Erlbaum Associates; 1993.

35. Saghaei, M. Random allocation software for parallel group randomized trials. BMC Med Res Methodol. 2004;4:26.

36. Milgram P. A spectacle-mounted liquid-crystal tachistoscope. Behav Res Methods Instrum Comput. 1987;19:449-456.

37. Manly T, Robertson IH, Galloway M, Hawkins K. The absent mind: further investigations of sustained attention to response. Neuropsychologia. 1999;37:661-670.

38. Manly T, Owen AM, McAvinue L, et al. Enhancing the sensitivity of a sustained attention task to frontal damage: convergent clinical and functional imaging evidence. Neurocase. 2003;9:340-349.

39. Dockree PM, Kelly SP, Roche RA, Hogan MJ, Reilly RB, Robertson IH. Behavioural and physiological impairments of sustained attention after traumatic brain injury. Brain Res Cogn Brain Res. 2004;20:403-414.

40. Robertson IH, Ward T, Ridgeway V, Nimmo-Smith I. The Test of Everyday Attention. Bury St Edmunds, UK: Thames Valley Test Company; 1994.

41. Crawford JR, Sommerville J, Robertson IH. Assessing the reliability and abnormality of subtest differences on the Test of Everyday Attention. Br J Clin Psychol. 1997;36:609-617.

42. Mangione CM, Lee PP, Gutierrez PR, Spritzer K, Berry S, Hays RD. Development of the 25-item National Eye Institute Visual Function Questionnaire. Arch Opthalmol. 2001;119:1050-1058 